\title{
The invisible support
}

\author{
The contribution of partners and families to scientists' work is often overlooked. It should be acknowledged and \\ supported more.
}

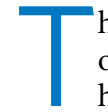
he lone genius working tirelessly on the research questions they have devoted their life to remains a prominent image in our culture, both within and outside of academia. Portraits of such characters therefore tend to underline their dedication, perhaps through endearing anecdotes of absent-mindedness in the face of real-world chores such as housework and childcare. When looked at from a different point of view, however, they also remind us of the often-unacknowledged support provided by scientists' partners and families that makes such absorption possible in the first place.

The nature of an academic career requires great dedication, and in practice this often means that the presence of such support is taken for granted, even by the researcher themselves.

Inevitably, this issue is embedded in our social conventions and the manner in which our public and private spheres intersect. Consider how the neuroscience pioneer Santiago Ramon y Cajal viewed the issue over a century ago: "for the man of science, the aid of a wife is just as necessary in youth as in old age", he wrote in his Advice for a Young Investigator. To him (and, no doubt, to most people in Europe at the time), a good wife was essential for running the household and taking care of the children so that the husband could pursue his scientific discoveries "free from anxiety". Ramon y Cajal explicitly stated his belief that, because of her sacrifice, the wife also deserved the fame derived from a scientific discovery, but the point is that for many women, especially in the past, this kind of support was all they were ever allowed or able to provide and they did indeed make a difference.

Most societies have undergone profound changes since the time of Ramon y Cajal, and with this we have seen a pronounced shift in the role that wives and partners can play in science and indeed in society as a whole, at least in principle: for one thing, women can choose to pursue a career in research. Yet we continue to grapple with the tensions between what is possible in principle, and what is doable in practice.

For example, the way support is distributed in families continues to be split across gender lines. Preliminary evidence shows that women have been

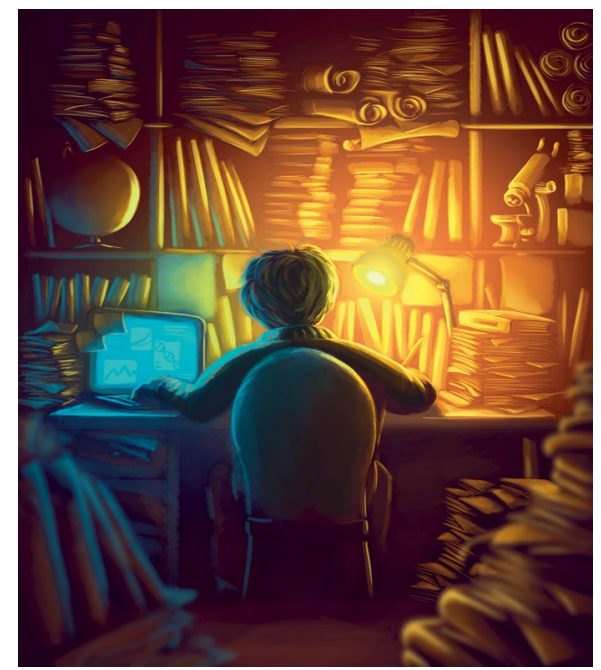

Credit: Falco Falcrow

disproportionately affected by the lockdowns put in place as part of the response to the COVID-19 pandemic (Viglione, G. Nature https://doi.org/ dwdw; 2020), with pre-existing inequalities in the distribution of house work and care responsibilities exacerbated. It is therefore no wonder that women in such circumstances are unable to remain as productive with their work, and further down the line this is likely to have an impact on job and promotion applications. To take this into account, the condensed-matter physicist Athene Donald has proposed that researchers with caring responsibilities should be able to claim an allowance of one paper or book chapter for every month of lockdown (Donald, A. Occam's Typewriter https://go.nature.com/2zOE5HG; 2020). Considering personal circumstances in this way could be an initial step to mitigate the adverse effects that the pandemic is likely to have on individuals' careers.

Looking beyond the current crisis, it seems clear that achieving an equal distribution of support between genders can only come with the cooperation of men. Societal expectations for men to be career-driven and for women to be carers are still strong. But if more men are willing to take on a primary parental role and share the mental load of housework, this can set an example and gradually change the expectation that childcare and chores are predominantly women's issues. These changes can only happen with the help of universities - and, for that matter, all employers - which can and should play a role in recognizing the issue by implementing helpful and enlightened policies.

For example, academics with childcare responsibilities can also benefit from small grants that pay for a carer or partner to travel with them to a conference. In one instance that gained attention on social media, Alicia Kraay, a postdoctoral researcher then based at the University of Michigan, was able to attend and chair a session at a conference shortly after having a child, after her institution paid for her husband's travel so that he could look after their child and she was able to nurse in between talks.

A common expectation is for academics to be able to move across the world, especially early in their careers. For the researcher with a partner this means choosing between leaving the family and network of support behind or moving together, which can come at a high personal cost for their partner and is likely to impact their career. One small way to address the latter issue is to fly both the researcher and their partner for the job interview. When researcher Kaitlin Cook applied for a faculty position at the Facility for Rare Isotope Beams at Michigan State University, the hiring institution paid for her wife's travel allowing her to get a sense for her career options and a feel for the town.

The products of house labour, childcare and emotional support are a lot less visible compared to a scientific publication or a grant award. Yet they are a crucial component to any researcher's life. Some will have mostly benefited from that support, whereas others will have dedicated huge parts of their lives providing it to others. Acknowledging the value and importance of this support and recognizing that there are differences in how it is (and indeed, could be) distributed is the first step for a fairer and altogether more inclusive academic environment.

Published online: 8 June 2020 https://doi.org/10.1038/s41567-020-0941-6 